\title{
Caracterización del profesorado de biología participante en la VII Olimpiada Costarricense de Ciencias Biológicas: Una mirada de sus estudiantes
}

\section{Characterization of the Biology Teachers Who Participated in the VII Costa Rican Olympiad of Biological Sciences: A Glance of Their Competitor's Students}

\author{
Federico Herrera \\ federico.herrera.madrigal@una.cr \\ Escuela de Ciencias Biológicas \\ Laboratorio de Enseñanza de las Ciencias, Universidad Nacional \\ Heredia, Costa Rica \\ José Pereira-Chaves \\ jose.pereira.chaves@una.cr \\ Escuela de Ciencias Biológicas \\ Laboratorio de Enseñanza de las Ciencias, Universidad Nacional \\ Heredia, Costa Rica \\ Nelson Muñoz-Simon \\ nelson.munoz.simon@una.cr \\ Escuela de Ciencias Biológicas \\ Laboratorio de Enseñanza de las Ciencias, Universidad Nacional \\ Heredia, Costa Rica
}

Recibido-Received: 13/dic/2015 / Corregido-Corrected: 4/oct /2016.

Aceptado-Accepted: 8/oct/2016 / Publicado-Published: 31/jul /2017.

\begin{abstract}
Resumen
Este estudio busca conocer aquellas fortalezas y oportunidades de mejora que se tiene en torno al proceso de enseñanza y aprendizaje de las ciencias biológicas en el aula y, por ello, conocer la percepción del estudiantado resulta fundamental. Se genera una línea base que refleja la situación actual del proceso de enseñanza-aprendizaje, con el fin de identificar las acciones educativas que se deben seguir potenciando y aquellas que se deben mejorar desde nuestra labor docente. Se presenta una caracterización de docentes de biología que participaron en VII Olimpiada Costarricense de Ciencias Biológicas, 2013, a partir de los aportes de sus estudiantes. Los resultados obtenidos, mediante la implementación de una encuesta, fueron positivos ya que un $83 \%$ de docentes obtuvo una alta calificación en su desempeño docente.
\end{abstract}

Palabras claves: Costa Rica; enseñanza de las ciencias; OLICOCIBI, desempeño docente, proceso de enseñanza-aprendizaje. 
UNICIENCIA Vol. 31, No. 2, pp. 83-97. Julio-diciembre, 2017.

ISSN Electrónico: 2215-3470

URL: www.revistas.una.ac.cr/uniciencia

DOI: http://dx.doi.org/10.15359/ru.31-2.6

Email: revistauniciencia@una.cr

\begin{abstract}
This study aims to understand the strengths and areas of improvement on the teaching and learning process of biological sciences within the classroom; therefore, knowing the perception of students is fundamental. It is generated a baseline that reflects the current situation of the teaching-learning process in order to identify the educational actions that could be further enhanced, and those that need improvement from the teaching perspective. A characterization of the biology teachers who participated in VII Costa Rican Biology Olympiad, 2013, is presented from their students' perspectives. The results obtained, through the implementation of a survey, were positive since $83 \%$ of teachers scored a high qualification in their teaching performance.
\end{abstract}

Keywords: Costa Rica; science teaching; OLICOCIBI, teaching performance, teaching-learning process, Biology Olympiads.

Desde su creación en el año 2007, el proyecto Olimpiadas Costarricenses de Ciencias Biológicas (OLICICOBI) se ha dado a la tarea de realizar los certámenes nacionales de biología, cuya dinámica e historia es explicada por Pereira-Chaves, Camacho-Vargas \& Muñoz-Simon (2013), así como a participar en justas internacionales de biología, llevar a cabo eventos de capacitación y recopilar información sobre el proceso de enseñanza-aprendizaje de la biología, mediante entrevistas y encuestas (Pereira-Chaves, 2010).

Al mismo tiempo, la OLICOCIBI ha buscado conocer aquellas fortalezas y oportunidades de mejora que se tienen en torno al proceso de enseñanza y aprendizaje de las ciencias biológicas $y$, por ello, conocer la percepción estudiantil es fundamental. En Costa Rica las iniciativas evaluadoras hacia el profesorado de biología, por parte de sus estudiantes de educación media, son prácticamente nulas. Por ende, con este estudio, se pretende generar una línea base que refleje la situación actual del proceso de enseñanza-aprendizaje con el fin de identificar las acciones educativas que se deben seguir potenciando y aquellas que se deben mejorar desde la labor docente. El objetivo del presente estudio es la caracterización, por parte de sus estudiantes, del personal docente de biología que participó en VII Olimpiada Costarricense de Ciencias Biológicas, celebrada en el 2013.

\title{
Marco teórico
}

Este estudio se encuentra enmarcado dentro del Proyecto OLICOCIBI, el cual se trata de una actividad de extensión universitaria, de la Universidad Nacional (UNA) cuya misión principal es el impulsar el interés por el estudio de las ciencias biológicas con el fin de contribuir al desarrollo científico y tecnológico del país, al promover el estudio activo, participativo y significativo de las ciencias biológicas dirigido a jóvenes estudiantes del III Ciclo de la Educación General Básica y del Ciclo Diversificado en las 27 direcciones regionales educativas del Ministerio de Educación Pública (MEP) a nivel nacional.

Este proyecto ha sido coordinado, desde su creación en el 2007, por la Universidad Nacional en Costa Rica (UNA). Un año después fue declarado de interés institucional debido a su impacto en el sistema educativo nacional, así como en la sociedad costarricense mediante el decreto SCU-159-2008 publicado en la Gaceta el 29 de febrero del 2008. Posteriormente el MEP, en el 2011, mediante el oficio DVM-AC-003888-2012, declaró las Olimpiadas Costarricenses de Ciencias Biológicas como una actividad de interés educativo, debido al impacto positivo que genera en la educación biológica costarricense. 
Actualmente, la comisión organizadora de OLICOCIBI, ahora interinstitucional, cuenta además con representantes del MEP, de la Universidad Estatal a Distancia (UNED), de la Universidad de Costa Rica (UCR), del Ministerio de Ciencia, Tecnología y Telecomunicaciones (MICITT), del Colegio de Licenciados y Profesores de la Enseñanza de las Ciencias, Filosofía y Letras (COLYPRO) y con el apoyo del Consejo Nacional para Investigaciones Científicas y Tecnológicas (CONICIT), el Consejo Nacional de Rectores (CONARE), por medio del Fondo Especial para el Financiamiento de la Educación Superior Estatal (FEES), y el Colegio de Biólogos de Costa Rica.

Respecto a la realización de la OLICOCIBI, la divulgación a nivel nacional es realizada en el mes de febrero mediante visitas a todos los colegios del país. También se realiza divulgación por medio de la página web de la OLICOCIBI, prensa escrita, boletines del MEP y COLYPRO, correos electrónicos, llamadas telefónicas y faxes a los centros educativos. Luego, en el mes de marzo, se realizan las inscripciones de estudiantes que competirán ya sea en la categoría A o la categoría $\mathrm{B}$, dependiendo el grado de secundaria en el que se encuentren.

Posteriormente, en el mes de mayo, se aplica a nivel nacional, en 13 recintos distribuidos por todo el país, un examen eliminatorio para ambas categorías. Durante el mes de junio, alrededor de un $10 \%$ de estudiantes es seleccionado para una segunda prueba, el examen final, en el recinto de la UNA, Heredia. Finalmente, estudiantes con los mejores resultados se convocan a participar en una preparación para representar al país en olimpiadas internacionales, como lo son la Olimpiada Iberoamericana de Biología (OIAB) y la Olimpiada Internacional de Biología (IBO), la cual es descrita por Pereira-Chaves et al. (2013).

De acuerdo con Pereira (2010), la enseñanza de las ciencias naturales a nivel de secundaria en Costa Rica es meramente teórica y en el país no se cuenta con estudios, en la enseñanza de la biología, que reflejen las vivencias en el aula.

Por lo tanto, este estudio viene a ser la primera evaluación estudiante-docente (de biología) que realiza el proyecto OLICOCIBI, lo que representa una línea base que refleja la situación actual del proceso de enseñanza de la biología y de cómo es el ambiente en el salón de clases. De esta manera será posible identificar las habilidades docentes que se deben reforzar mediante la actualización por medio de talleres y cursos de capacitación. Además, se pretende conocer los aspectos educativos en los que se debe trabajar aún más; así como las condiciones requeridas para incentivar la creatividad, la innovación y lograr que la enseñanza de las ciencias en el aula contribuya, de mejor manera, a la formación del pensamiento científico que se espera de la formación en el nivel de la educación secundaria.

\section{Metodología}

La investigación fuellevada a cabo a nivel nacional, en los 13 recintos disponibles, distribuidos a lo largo del país, para la aplicación del examen y abarcó las 27 direcciones regionales educativas del MEP, ubicadas en San José centro, Pérez Zeledón, Heredia, Nicoya, Liberia, Alajuela centro, San Ramón, Puntarenas centro, Ciudad Neilly, San Carlos, Cartago, Limón centro y Guápiles, por lo cual se toma en cuenta el contexto educativo de muchas instituciones, tanto rurales como urbanas, así como la multiplicidad de educandos que estudian en ellas. Específicamente, se contó con el apoyo de 143 estudiantes participantes de la VII OLICOCIBI, quienes fueron seleccionados de forma aleatoria para responder un cuestionario aplicado inmediatamente después de realizar el Examen Eliminatorio de la VII OLICOCIBI. Esto representa una muestra 
UNICIENCIA Vol. 31, No. 2, pp. 83-97. Julio-diciembre, 2017.

de un $10.4 \%$ de los 1.373 estudiantes provenientes de 180 colegios, tanto privados como públicos, que participaron en dicha olimpiada.

El estudio se organizó desde un enfoque cuantitativo. El instrumento suministrado fue de tipo simple o auto-administrado, en el cual no hubo intervención directa de ninguna persona más que de cada estudiante quien contestaba por escrito. La estructura de este contó con 49 preguntas divididas en 8 aspectos principales a evaluar del personal docente de biología, los cuales fueron:

1. Dominio de los contenidos

2. Adecuación de metas y objetivos

3. Uso de material de apoyo

4. Uso de diferentes métodos de enseñanza

5. Fomento del trabajo en grupo

6. Respeto hacia la participación de estudiantes

7. Actualización de los contenidos

8. Papel de guía durante el proceso de enseñanza

De las preguntas se tomó la interrogante más significativa, por aspecto, que se deseaba evaluar sobre el personal docente de biología de educación media al impartir sus lecciones de biología. dichas preguntas fueron:

1. ¿Domina el profesor los contenidos?

2. ¿Planifica el tema (lección)?

3. ¿Presenta de manera atractiva la información?

4. ¿Realiza prácticas, experimentos, giras o actividades similares?

5. ¿Hace preguntas para fomentar la participación del grupo?

6. ¿Es respetuoso con la participación y los comentarios de los estudiantes?

7. ¿Resalta nuevas investigaciones en el área de biología?

8. ¿Motiva al estudiantado a participar en eventos extracurriculares (i.e. olimpiadas)?

A su vez, los descriptores utilizados para responder dicha encuesta fueron los propuestos por Likert (1932):

- Totalmente en desacuerdo (1)

- En desacuerdo (2)

- Indiferente (3)

- De acuerdo (4)

- Totalmente de acuerdo (5) 
El instrumento utilizado fue validado mediante su aplicación a diez docentes de biología que han participado en las olimpiadas, en ediciones anteriores, y a 15 estudiantes seleccionados para los entrenamientos, como posibles candidatos para representar a Costa Rica en justas internacionales, cuyo objetivo fue, con sus observaciones, verificar su comprensión e intencionalidad, para lo cual se consideraron las observaciones y aportes de todos los grupos colaboradores.

Finalmente, los resultados de la encuesta fueron analizados con el software SPSS ${ }^{\circledast}$ (Statistical Package for the Social Sciences). Por su parte, el análisis de los resultados se llevó a cabo con base en la estadística descriptiva, con la cual se obtuvieron las frecuencias para cada pregunta del cuestionario.

\section{Resultados y análisis}

Los resultados mostrados a continuación fueron seleccionados por su pertinencia para el objetivo principal de la investigación, cuyo carácter es exploratorio. Cada figura muestra las respuestas para las interrogantes clave.

A continuación, se presentan los resultados obtenidos para la inter rogante sobre el dominio de los contenidos del curso por parte del personal docente de biología, correspondiente al aspecto Dominio de los contenidos (ver figura 1). Se evidencia que el $70 \%$ de estudiantes está totalmente de acuerdo con que el profesorado posee un dominio adecuado sobre los contenidos, por su parte, un $20 \%$ está de acuerdo con este aspecto. Cerca del $8 \%$ de estudiantes se mantuvo indiferente a las capacidades de su docente. Finalmente, cerca del 1\% del alumnado está en desacuerdo con que el profesorado domine los contenidos de la materia, mientras que nadie de la población estudiada está totalmente en desacuerdo con que su docente domine los contenidos de la materia de biología.

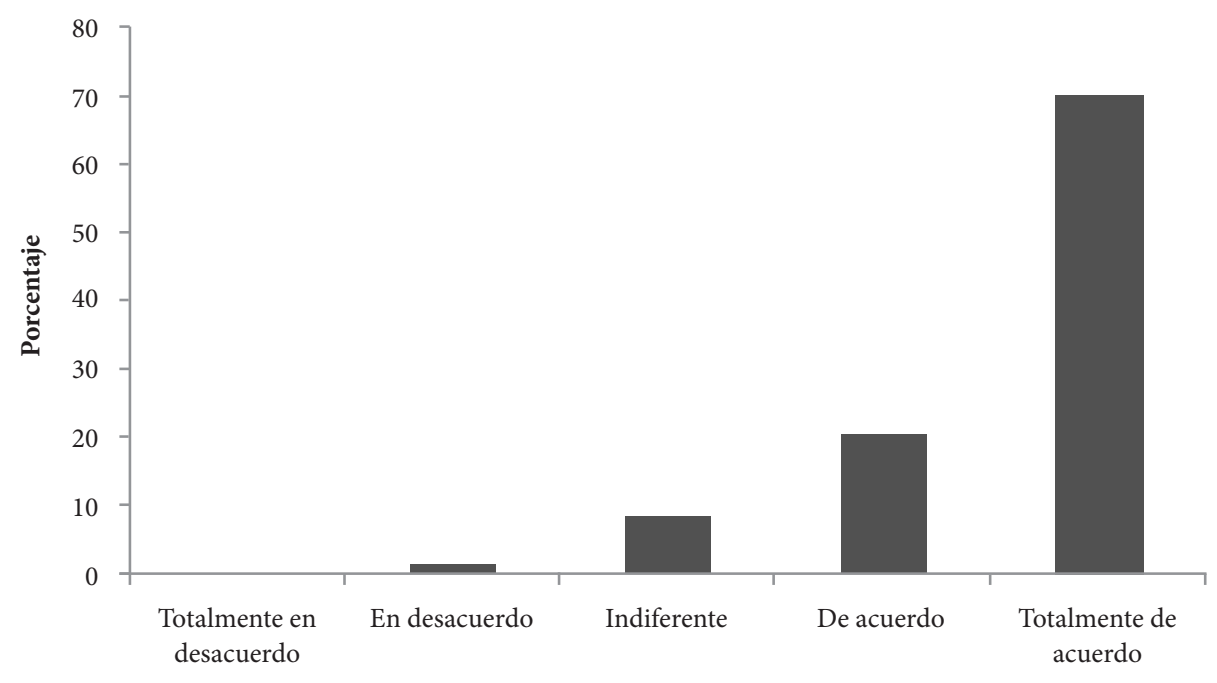

Figura 1. Resultado de la encuesta acerca del Dominio de los contenidos por parte del profesorado de biología, aplicado a estudiantes participantes de la VII OLICOCIBI 2013 ( $n=143$ ). Datos propios de la investigación. 
UNICIENCIA Vol. 31, No. 2, pp. 83-97. Julio-diciembre, 2017.

En relación con el dominio de docentes de biología sobre la materia que imparten, se puede notar que la mayoría tiene la capacidad para reflejar dicho conocimiento en sus estudiantes. El 90\% de estudiantes, que están totalmente de acuerdo y de acuerdo, proporcionan cifras que permiten conocer y perder un poco la preocupación que se tiene sobre las capacidades docentes en esta área, lo cual vendría a reflejar el alto porcentaje de estudiantes que perciben un total dominio del contenido por parte del profesorado que labora a nivel nacional. Por otra parte, lo ideal sería lograr cambiar los porcentajes de estudiantes restantes, un 9\%, totalmente de acuerdo y de acuerdo, lo cual es meta en la enseñanza de la biología. Sin embargo, el no tener estudiantes que estén totalmente en desacuerdo con el dominio de su docente sobre los contenidos, refleja que en realidad la labor docente posee un alto índice de calidad y no es un factor alarmante que necesite ser tratado con urgencia.

A una conclusión similar llegaron Del-Pozo, Fernández, González \& de Juanas (2013) en una encuesta similar, pero aplicada a profesorado en Madrid, España. En ese estudio, el 79\% consideró fundamental dominar los contenidos del área que imparte ya que así se garantiza que se puedan transmitir los conocimientos correctamente al alumnado.

A manera de recomendación para elevar este porcentaje de $9 \%$, de las dos categorías más bajas, sería una mayor preocupación por parte del personal docente de tomar cursos de capacitación y de actualización para que desarrolle mejores herramientas para desempeñar su trabajo. Por otro lado, Paniagua (2004) señala quelosesfuerzos de actualización docente que realizael MEP son insatisfactorios, onerosos, con bajo o ningún impacto en la actividad del aula, además, resalta que el MEP no cuenta con un sistema formal de detección de necesidades. Esta situación es bastante preocupante, ya que la capacitación docente y permanente actualización les permite a docentes promover la necesidad del cambio, preparar al estudiantado para enfrentar con criticidad y creatividad los cambios acelerados en los campos tecnológicos, científicos, sociales, económicos y culturales en esta nueva era globalizada. De la misma manera, esto contribuye a desarrollar metodologías participativas que favorezcan los aprendizajes significativos, potenciar el uso de diferentes recursos educativos, promover una renovación metodológica en las diferentes áreas del quehacer docente, entre otros (Herdoiza, 2004).

Por otro lado, con respecto al apartado sobre Adecuación de metas y objetivos, los resultados obtenidos para la interrogante de si el personal docente de biología planificaba las lecciones (ver figura 2), fueron que casi el $57 \%$ del total de estudiantes estaba totalmente de acuerdo con que su docente planifica las lecciones, un $29 \%$ estuvo de acuerdo; mientras que un $12 \%$ de estudiantes se anotó como indiferente, este grupo, junto a las dos categorías más bajas, suma un 15\%.

En relación con la interrogante planteada, se puede notar que el estudiantado está totalmente de acuerdo y de acuerdo en que su profesorado planea las lecciones, lo cual representa casi un $86 \%$ del total, situación que deja claro que el personal docente lleva a cabo su trabajo de forma adecuada, estructurando los contenidos de manera tal que se pueda generar un aprendizaje efectivo en sus estudiantes. Por su parte, el porcentaje de estudiantes en desacuerdo y totalmente en desacuerdo refleja que es necesario hacer algo para cambiar esta situación, debido a que parte de lo que significa ser docente es llevar las lecciones previamente preparadas para evitar generar confusiones o pérdidas de tiempo en sus estudiantes; no obstante, dicho porcentaje es del 3\% del total, por lo que la calidad en educación de la biología se mantiene en niveles altos. Por su parte, Nérici (1969) establece que, en cuanto a la planificación de las lecciones, el maestro o maestra tiene una responsabilidad moral, económica y ética de planificarlas, así como priorizar los contenidos para aumentar la eficiencia de la enseñanza, evitar improvisaciones que confundan al alumnado, proporcionar secuencia y progresividad a los trabajos escolares, así como mostrar consideración y respeto hacia sus estudiantes. 
ISSN Electrónico: 2215-3470

DOI: http://dx.doi.org/10.15359/ru.31-2.6
UNICIENCIA Vol. 31, No. 2, pp. 83-97. Julio-diciembre, 2017.

URL: www.revistas.una.ac.cr/uniciencia Email: revistauniciencia@una.cr

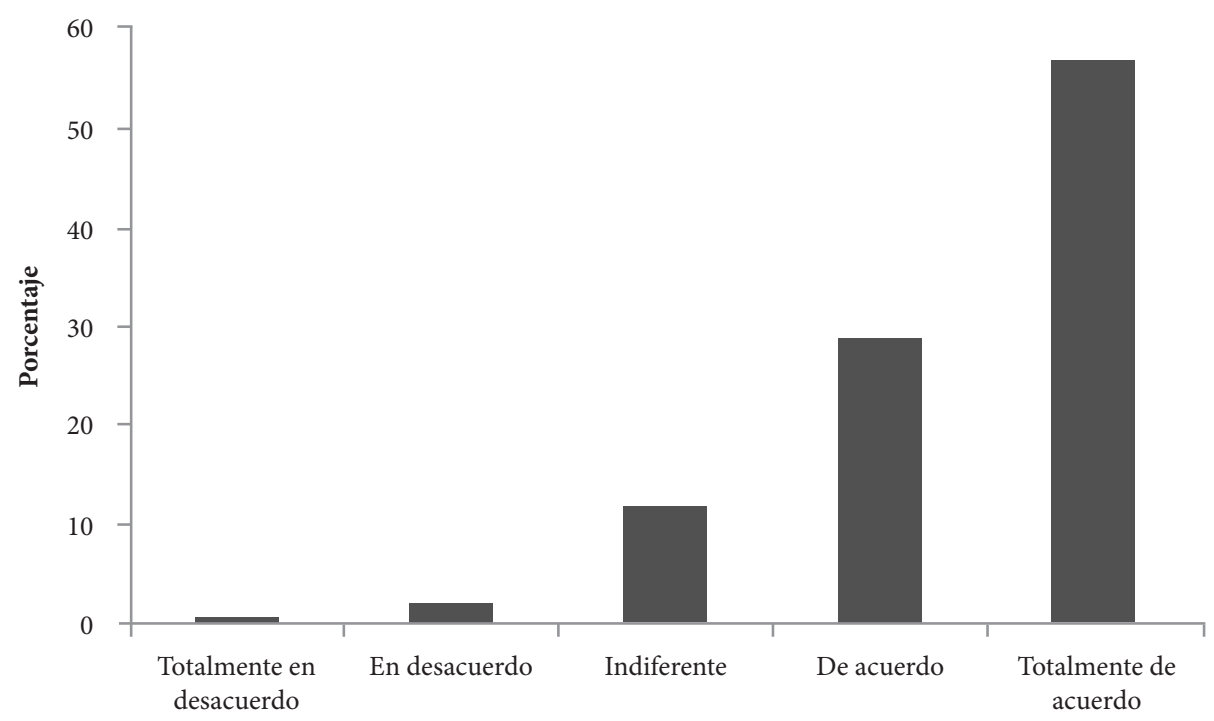

Figura 2. Resultado de la encuesta acerca de la Planificación de las lecciones por parte de sus docentes de biología, aplicado a estudiantes participantes de la VII OLICOCIBI $2013(\mathrm{n}=143)$. Datos propios de la investigación.

En cuanto al aspecto de si su docente de biología realiza presentaciones atractivas, correspondiente al apartado Uso de materiales de apoyo, los resultados obtenidos fueron que, cerca del $52 \%$ de estudiantes está totalmente de acuerdo con que realiza presentaciones atractivas, además, un $29 \%$ que asegura estar de acuerdo con esto (figura 3). Solamente un $12 \%$ de estudiantes es indiferente a la cuestión planteada. Finalmente, un $7 \%$ está en desacuerdo con que su profesor o profesora realiza presentaciones atractivas, mientras que cerca del $1 \%$ asegura estar totalmente en desacuerdo.

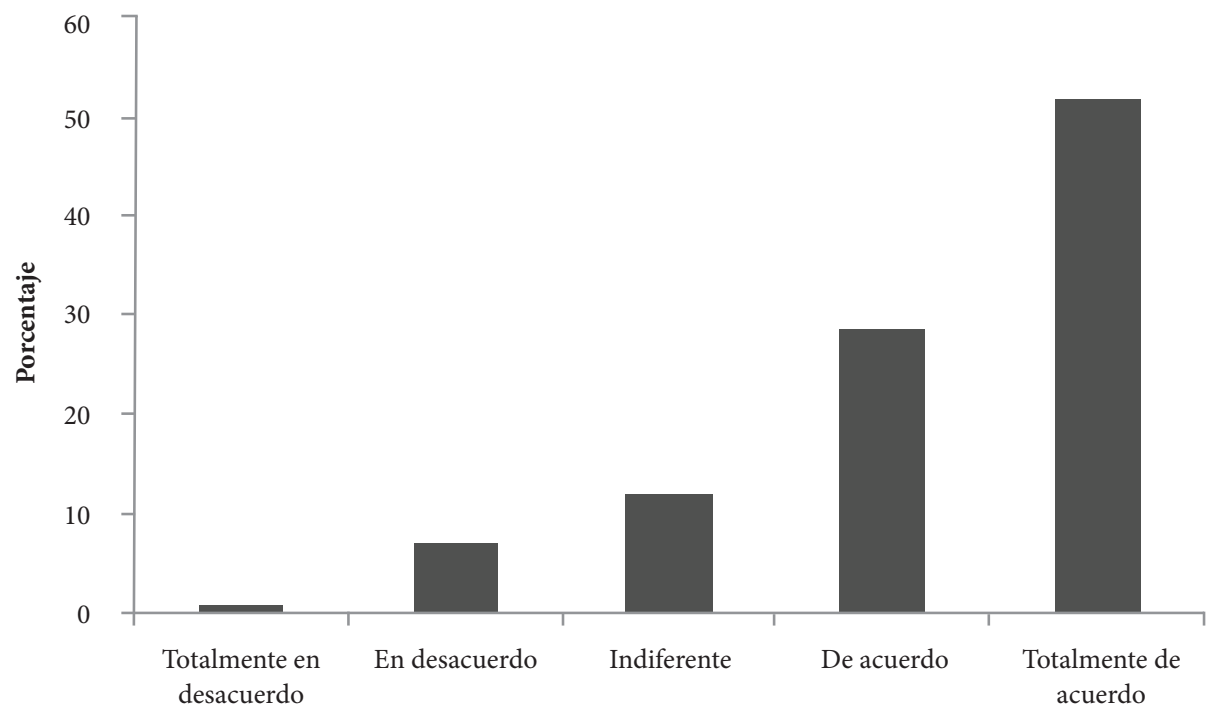

Figura 3. Resultado de la encuesta acerca del atractivo de las presentaciones realizadas por sus profesores de biología, aplicado a estudiantes participantes de la VII OLICOCIBI $2013(\mathrm{n}=143)$. Datos propios de la investigación. 
UNICIENCIA Vol. 31, No. 2, pp. 83-97. Julio-diciembre, 2017.

En relación con el material de apoyo, se tomó en consideración principalmente a las diapositivas como forma factible de presentar la materia al estudiantado. De esta manera, se puede observar que un $81 \%$ de estudiantes está totalmente de acuerdo y de acuerdo con que el recurso utilizado no solo es adecuado, sino que también es atractivo a la hora de recibir clases. No obstante, existe un porcentaje considerable que considera no atractiva dicha metodología, por lo que están indiferentes, en desacuerdo o totalmente en desacuerdo con que sea un recurso atrayente y motivante. Este porcentaje de estudiantes inconformes es del $20 \%$ del total, lo que hace un llamado a la adaptación de presentaciones más atractivas e interesantes que permitan un mejor desarrollo y aprendizaje del alumnado en la materia de biología, tema de gran interés para la OLICOCIBI.

Continuando con lo atractivo de las presentaciones, es importante que el personal docente promueva espacios de aprendizaje más interactivos, ya que actualmente vivimos en una era digital en donde la mayoría de estudiantes domina las tecnologías de la información y la comunicación (TIC) que les facilitan acceso a la información y a formas alternativas de representar el conocimiento. Son personas que viven la cultura del internet (Domingo \& Fuentes, 2010). Por eso es de suma importancia que el profesorado implemente estas tecnologías en el aula para hacer sus lecciones más atrayentes, ya que, según Bravo-Ramos (2005), el 80\% de las presentaciones realizadas por profesionales se lleva a cabo utilizando medios visuales. Almenara (1998) y Bravo-Ramos (2005) proponen las respectivas pautas o características que debe poseer una presentación para que sea atractiva y acorde con la audiencia.

Con respecto a la implementación de prácticas, experimentos o giras por parte del profesorado de biología, correspondiente al apartado Uso de diferentes métodos de enseñanza, se obtuvo que casi un $64 \%$ de estudiantes se encontraba totalmente de acuerdo y de acuerdo con que su docente de biología realizaba actividades alternativas para enseñar la materia como prácticas, experimentos y giras de campo. Mientras que lo contrario ocurre con un $20 \%$ de estudiantes quienes estaban indiferentes al respecto y con un $16 \%$ que se encontraban totalmente en desacuerdo y en desacuerdo (ver figuraa 4).

Estas últimas cifras reflejan una situación preocupante, ya que el trabajo experimental y de campo es una parte fundamental de las disciplinas científicas y es debido a esto que la OLICOCIBI implementa pruebas prácticas en sus exámenes finales del certamen nacional. Es lógico pensar que la falta de dichos espacios prácticos en muchas instituciones o centros educativos podría deberse a la falta de recursos, como tiempo, materiales, laboratorios, logística, entre otros. Sin embargo, López-García \& Morcillo-Ortega (2007) proponen laboratorios virtuales, disponibles en línea, como alternativas a estas situaciones. En ellas, el estudiantado puede realizar simulaciones de biología general, laboratorios virtuales de biología en español, prácticas de microscopía, disecciones, acceder a colecciones virtuales, entre otros. El objetivo de dichas prácticas de laboratorio, presenciales o virtuales, es que posean un efecto motivador, que familiaricen al estudiantado con la metodología científica y promuevan el aprendizaje de conceptos científicos (Bastida de la Calle, Ramos-Fernández \& Soto-López, 1990).

De igual manera, Lemke (2006) indica que las giras de campo son importantes para el aprendizaje de las ciencias y la aplicación de estas a la realidad, como podría ser la visita a laboratorios, fábricas, centrales eléctricas, zoológicos, acuarios, museos, entre otros. Por otro lado, según Walkerdine, en Lemke (2006), la educación científica se ha vuelto demasiado aislada de las cuestiones de la vida cotidiana, puesto que existe la tendencia a pensar que el aprendizaje abstracto es más noble que el aprendizaje práctico y concreto. 
ISSN Electrónico: 2215-3470 DOI: http://dx.doi.org/10.15359/ru.31-2.6
UNICIENCIA Vol. 31, No. 2, pp. 83-97. Julio-diciembre, 2017.

URL: www.revistas.una.ac.cr/uniciencia Email: revistauniciencia@una.cr

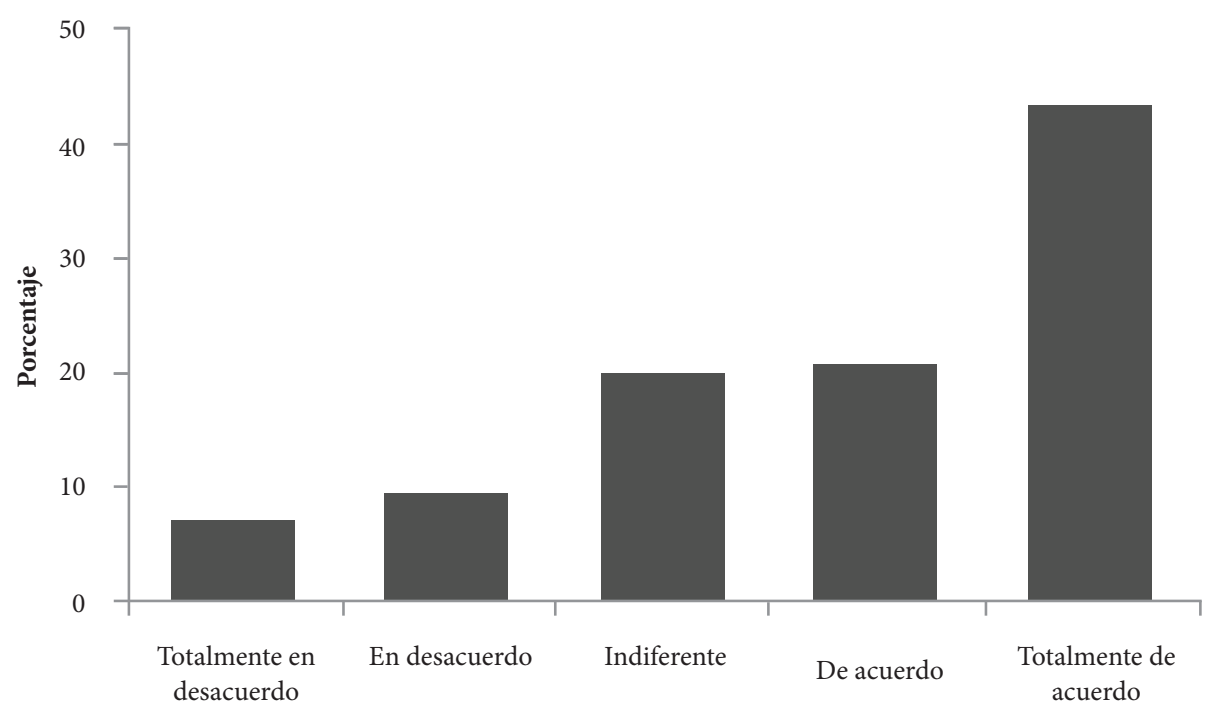

Figura 4. Resultado de la encuesta acerca de la implementación de prácticas, experimentos o giras por parte del profesorado de biología, aplicado a estudiantes participantes de la VII OLICOCIBI $2013(\mathrm{n}=143)$. Datos propios de la investigación.

Con respecto a la generación de preguntas que fomenten la participación del grupo por parte del profesorado de biología, correspondiente al apartado Fomento del trabajo en grupo (figura 5), se obtuvo que solamente un 5\% estuvo totalmente en desacuerdo y en desacuerdo, al sugerir que su profesor o profesora de biología no realizaba preguntas que motivaran la participación del grupo, mientras que el $86 \%$ estuvo de acuerdo y totalmente de acuerdo.

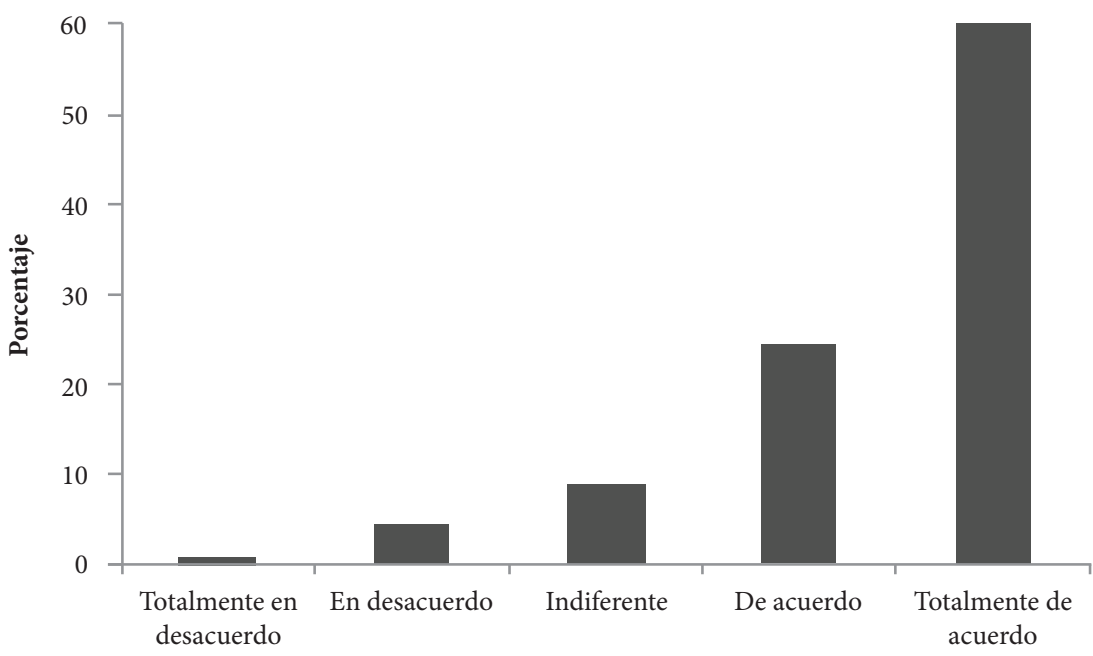

Figura 5. Resultado de la encuesta acerca de la implementación de generación de preguntas para fomentar la participación en el aula por parte del profesorado de biología, aplicado a estudiantes participantes de la VII OLICOCIBI $2013(n=143)$.

Datos propios de la investigación. 
Lo anterior refleja que en las lecciones de biología se da una muy buena interacción entre docente y su alumnado, ya que enseñar y aprender es, básicamente, un proceso de comunicación entre estudiantes, profesorado y conocimiento, y en este proceso las preguntas cumplen un papel principal (Márquez-Bargalló \& Roca-Tort, 2006).

De este modo, al plantear interrogantes se verifica que el estudiantado haya entendido las explicaciones o demostraciones y se asegura que progrese de manera adecuada hacia el logro de los objetivos de aprendizaje. ITESM (1999) estipula que el método de preguntas sirve para guiar al estudiantado a la discusión y análisis de la información pertinente a la materia. De esta manera se estimula el pensamiento crítico, se promueven habilidades para el análisis y síntesis de la información. Esto es importante de recalcar, ya que se evidencia que ha habido un cambio en la dinámica de enseñanza-aprendizaje y en la interacción docente - estudiante y viceversa, lo cual difiere con lo planteado por Quinquer (2004) $)_{2}$ quien afirmaba que la mayoría de las clases de ciencias en bachillerato se daban mediante clases expositivas donde el personal docente era el centro de la actividad. Por lo tanto, es vital que se elaboren y discutan preguntas relacionadas con la materia para promover el diálogo y comentar las respuestas brindadas por el estudiantado.

Continuando con los resultados obtenidos por medio de la encuesta, para la interrogante de si el profesorado muestra una actitud de respeto hacia la participación y comentarios de sus estudiantes, correspondiente al apartado sobre Respeto hacia la participación de estudiantes se obtuvo lo siguiente (figura 6): Es posible observar que hay un gran número de estudiantes, un 90\%, que está totalmente de acuerdo y de acuerdo con que su profesor o profesora muestra respeto por sus participaciones y comentarios en el aula. Mientras que el porcentaje de estudiantes que admite estar indiferentes, en desacuerdo y totalmente en desacuerdo, suman un $10 \%$ del total encuestado.

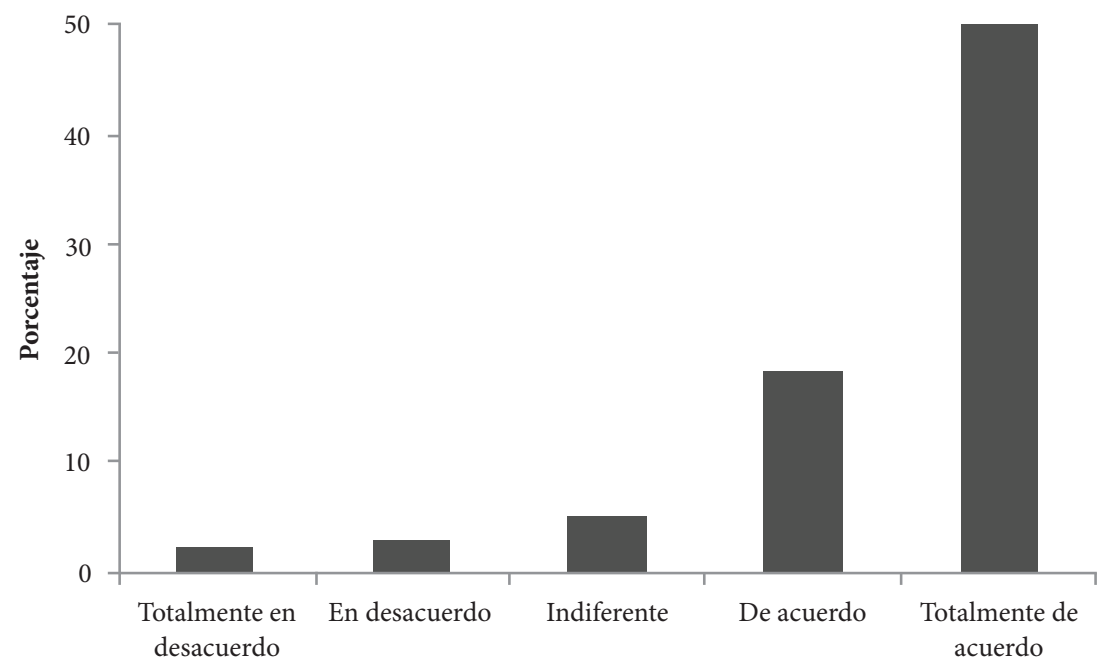

Figura 6. Resultado de la encuesta acerca del respeto dado hacia la participación y comentarios de estudiantes por parte de sus docentes de biología, aplicado a estudiantes participantes de la VII OLICOCIBI $2013(\mathrm{n}=143)$. Datos propios de la investigación. 
Aunque el resultado sea que un 90\% está totalmente de acuerdo y de acuerdo, es inquietante ya que significa que la diferencia no siente ese respeto de parte del profesor o profesora, el cual es sumamente vital, ya que este último esta figura está encargada promover el clima del aula, el cual es descrito por Marchena, en Barreda-Gómez (2012, p. 5) como "una construcción originada por las relaciones sociales que entablan los protagonistas de una clase así como por la forma de pensar de cada uno de ellos". En otras palabras, es el personal docente es el encargado de crear un ambiente ideal en el aula donde se fomente, en sus discentes, sentimientos de superación, de valor personal, de confianza, de mutuo respeto a las opiniones o formas de pensar y de formación de valores y actitudes que les lleven a querer saber más sobre el estudio de esta materia. De acuerdo con Berzal \& Ortíz (2006), el personal docente es el principal motor de inspiración y, sobre todo, de motivación para sus estudiantes, y de él depende que se potencie o no al sujeto aprendiente.

Siguiendo con la sección de actualización de contenidos por parte del personal docente, una de las preguntas que se le planteó al estudiantado fue si el docente resaltaba en el salón de clases nuevas investigaciones en el área de la biología (figura 7). Se obtuvo que un $2 \%$ estuvo totalmente en desacuerdo y en desacuerdo contrastado con un $84 \%$ de estudiantes que estuvo de acuerdo y totalmente de acuerdo con que su docente de biología sí resaltaba investigaciones recientes dentro del salón de clase.

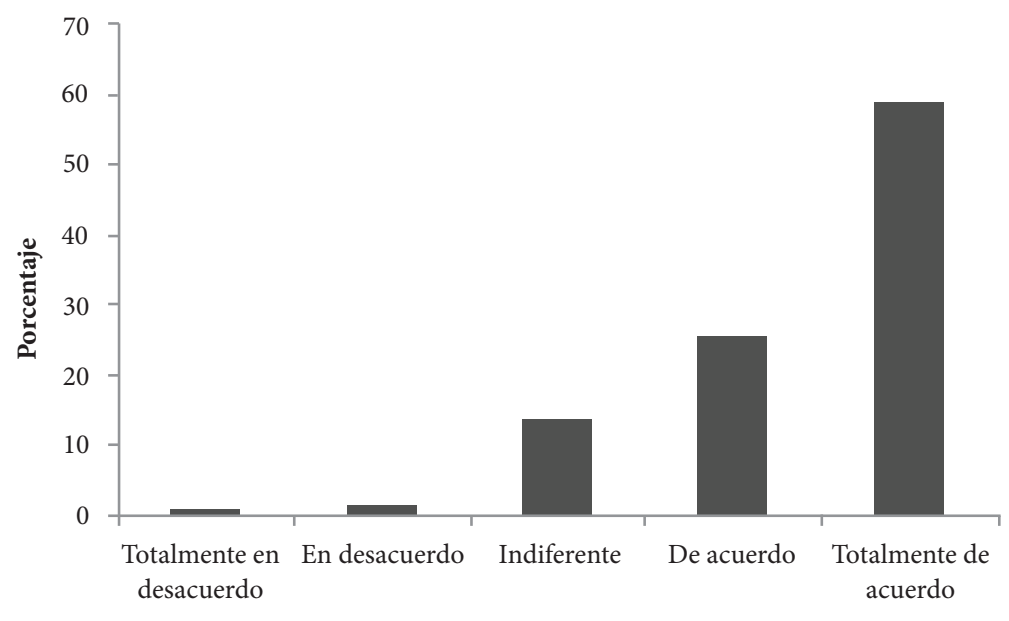

Figura 7. Resultado de la encuesta acerca de si el personal docente resalta nuevas investigaciones en el área de la biología, aplicado a estudiantes participantes de la VII OLICOCIBI 2013 ( $\mathrm{n}=143$ ). Datos propios de la investigación.

Este resultado es muy positivo, ya que se evidencia que el personal docente se informa sobre nuevas investigaciones, lo cual contribuye significativamente a mejorar no solo su actividad docente sino también a adaptar a la realidad la materia para su alumnado.

De este mismo modo, la utilización de nuevas investigaciones en el campo de la biología les permite tanto a estudiantes como al profesorado mantenerse al día y tener a mano la información más actualizada. Una manera de lograr esto podría ser utilizar la prensa escrita, como periódicos, revistas especializadas o artículos científicos, como medio alternativo a los textos escolares, no para sustituirlos sino para complementar con información e investigaciones y problemáticas actuales. Por lo general, estos escritos son muy poco utilizados. 
UNICIENCIA Vol. 31, No. 2, pp. 83-97. Julio-diciembre, 2017.

De acuerdo con Ramírez-Silva, Addine-Fernández \& Addine-Ramírez (2010), la utilización de este tipo de información actualizada complementaria desarrolla en el estudiantado habilidades y actitudes personales como aprender a captar las ideas relevantes, analizarlas y proponer síntesis personales; saber argumentar puntos de vista; juzgar la información obtenida; desarrollar actitudes de colaboración, y relacionar las ideas.

Finalmente, para la sección papel de guía durante el proceso de enseñanza, una de las preguntas que se le planteó al estudiantado fue si el docente lo motivaba a participar en eventos o actividades extracurriculares, como la OLICOCIBI, por ejemplo (figura 8). Se obtuvo que solo un 5\% estuvo totalmente en desacuerdo y en desacuerdo mientras que un $88 \%$ estuvo de acuerdo y totalmente de acuerdo.

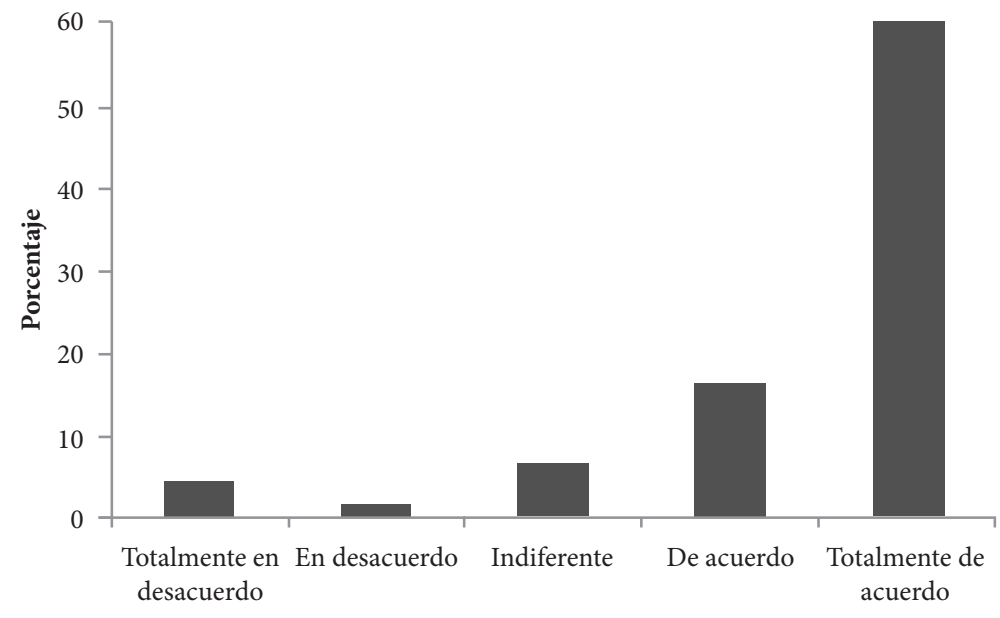

Figura 8. Resultado de la encuesta acerca de si el personal docente motiva a sus estudiantes a participar en actividades extracurriculares, aplicado a estudiantes participantes de la VII OLICOCIBI $2013(\mathrm{n}=143)$. Datos propios de la investigación.

Esta sección es de gran significancia directa para la OLICOCIBI. Acá vale la pena resaltar la importancia de que estudiantes participen en este tipo de actividades, ya que promueven el estudio activo, participativo y significativo de las ciencias biológicas a nivel de colegio y preuniversitario (Camacho-Vargas \& Pereira-Chaves, 2012). De acuerdo con Rodríguez-Calvo (2012), este tipo de competencias amplía los conocimientos biológicos; promueve el interés por el estudio de la biología; lleva al estudiantado a ser reflexivo, analítico, crítico, creativo, observador; propicia el intercambio cultural, social y cognitivo entre estudiantes de diversos centros educativos de todo el país, entre otros aspectos.

En resumen, para toda la encuesta, los resultados son positivos, y reflejan un alto desempeño por la parte de docentes de biología, ya que un $83 \%$ de estudiantes estuvo de acuerdo y totalmente de acuerdo con su profesor o profesora de biología y apenas solo un 5.5\% estaba en desacuerdo y totalmente en desacuerdo. 
ISSN Electrónico: 2215-3470

DOI: http://dx.doi.org/10.15359/ru.31-2.6
UNICIENCIA Vol. 31, No. 2, pp. 83-97. Julio-diciembre, 2017.

URL: www.revistas.una.ac.cr/uniciencia Email: revistauniciencia@una.cr

\section{Conclusiones y recomendaciones}

Se evidencia que el profesorado de biología que participó en la VII OLICOCIBI tiene un alto perfil profesional, ya que domina y presenta la materia de manera adecuada y atractiva, realiza preguntas para promover la participación del alumnado, es respetuoso ante sus comentarios e intervenciones, resalta nuevas investigaciones y prácticas de laboratorio o giras, con lo cual acerca al estudiantado a la realidad y lo motiva a que participe en actividades académicas extracurriculares.

Aunado a lo anterior, siempre es importante promover una mayor preocupación por parte del personal docente de tomar cursos de capacitación para que desarrolle mejores herramientas para desempeñar su trabajo. Desde su creación, OLICOCIBI ha estado realizando activamente esfuerzos adicionales para brindar talleres, clases y capacitaciones a docentes de biología, así como organizando simposios donde el personal docente pueda compartir sus investigaciones o experiencias. Entre las últimas capacitaciones que se han brindado a docentes de biología de educación media, por parte de OLICOCIBI, están: "Funciones y propiedades de los compuestos orgánicos de la célula" (40 horas, V-2014), "Actualización en anatomía y fisiología humana" (40 horas, XI-2014), "Herencia y mutaciones" (40 horas, VI-2015); "Fotosíntesis y ecología de la planta" (40 horas, VI-2015); "Fotosíntesis: importancia y aplicaciones actuales" (dos cursos simultáneos de 40 horas, X-2015) y las mencionadas por Rodríguez-Calvo \& Pereira-Chaves (2015) implementadas del 2008 al 2013. Entre los simposios organizados por OLICOCIBI están el I Simposio Costarricense de Enseñanza de Ciencias (30-I-2015) y el II Simposio de la Enseñanza de las Ciencias y la Biología (por celebrarse, 28-29-I-2016), ya que un objetivo de OLICOCIBI es ayudar al mejoramiento del proceso de enseñanza y aprendizaje mediante capacitaciones y actualizaciones en el área de las ciencias biológicas en secundaria (ECB, 2015).

Por otra parte, es necesario fomentar la utilización de las prácticas de laboratorio como un medio de motivación y de enseñanza más activa, en donde se impulse el método científico como generador de nuevo conocimiento y para que aprendan a razonar sistemáticamente. Además, se aumenta la comprensión de los aspectos tratados en la teoría y se motiva al estudiantado, ya que, de acuerdo con lo observado en la OLICOCIBI y lo comentado por el propio estudiantado, disfrutan cuando trabajan en el laboratorio.

Aunado a esto, una comunicación asertiva entre docente y estudiante es de suma importancia para fomentar y desarrollar la participación en el aula, ya que les permite, tanto a educadores y educadoras como a estudiantes, expresar sus propios sentimientos y opiniones, y saber que se les escucha y toma en serio.

En este mismo sentido, es importante inculcar en el alumnado la participación en actividades extracurriculares, pues estas propician la ampliación de los conocimientos biológicos; los hábitos de estudio; la responsabilidad; el intercambio social, cognitivo y cultural; así como el análisis, la creatividad y la observación.

Finalmente, sería interesante realizar estas mismas interrogantes a estudiantes que no participen o hayan participado en la OLICOCIBI para, con esa evaluación docente obtenida, evidenciar si existe alguna diferencia entre el profesorado que regularmente participa en este tipo de actividades extracurriculares y que regularmente atiende capacitaciones organizadas por la OLICOCIBI y docentes de biología que no lo hacen. 
UNICIENCIA Vol. 31, No. 2, pp. 83-97. Julio-diciembre, 2017.

ISSN Electrónico: 2215-3470

URL: www.revistas.una.ac.cr/uniciencia

DOI: $\underline{\text { http://dx.doi.org/10.15359/ru.31-2.6 }}$

Email: revistauniciencia@una.cr

\section{Referencias}

Almenara, J. (1998). Uso didáctico de las presentaciones colectivas por medios informáticos. Comunicar: Revista científica iberoamericana de comunicación y educación, 11, 149-157.

Barreda-Gómez, M. S. (2012). El docente como gestor del clima del aula. Factores a tener en cuenta. (Tesis inédita de maestría). Universidad de Cantabria. Santander, España.

Bastida de la Calle, M. F., Ramos-Fernández, F., \& Soto-López, J. (1990). Prácticas de laboratorio: ¿Una inversión poco rentable? Investigación en la Escuela, 11, 77-91.

Berzal, M., \& Ortiz, M. (2006). El papel de las reuniones científicas y educativas en la promoción de la investigación, la innovación y la formación del profesorado de biología. Revista de Educación en Biología, 9(1), 6-13.

Bravo-Ramos, J. L. (2005). Los gestores de presentaciones como apoyo en clases, ponencias y conferencias. Instituto de Ciencias de la Educación, Universidad Politécnica. Madrid, España.

Camacho-Vargas, S., \& Pereira-Chaves, J. (2012). Construcción de la dimensión cognitiva de alfabetización científica en el estudiantado, a través de las Olimpiadas Costarricenses de Ciencias Biológicas. Revista Electrónica Educare, 16(2), 217-236.

Del Pozo, R., Fernández, P., González, M., \& de Juanas, A. (2013). El dominio de los contenidos escolares: Competencia profesional y formación inicial de maestros. Revista de Educación, 360, 363-387.

Domingo, M., \& Fuentes, M. (2010). Innovación educativa: Experimentar con las TIC y reflexionar sobre su uso. Pixel-Bit. Revista de Medios y Educación, 36, 171-180.

ECB - Escuela de Ciencias Biológicas. (2015). Normativa Olimpiadas Costarricenses de Ciencias Biológicas (OLICOCIBI), Universidad Nacional, Heredia, Costa Rica.

Herdoiza, M. (2004). Capacitación docente. Strengthening Achievement in Basic Education (SABE) Project. Recuperado de http://pdf.usaid.gov/pdf_docs/PNACG311.pdf

ITESM - Instituto Tecnológico y Estudios Superiores de Monterrey. (1999). Capacitación en estrategias y técnicas didácticas. Recuperado de: http://sitios.itesm.mx/va/dide/documentos/inf-doc/ introduccion.htm

Lemke, J. (2006). Investigar para el futuro de la aplicación científica: Nuevas formas de aprender, nuevas formas de vivir. Enseñanza de las Ciencias, 24(1), 5-12.

Likert, R. (1932). A technique for the measurement of attitudes. Archives of Psychology, 22(140), 1-55.

López-García, M., \& Morcillo-Ortega, J. G. (2007). Las TIC en la enseñanza de la biología en la educación secundaria: Los laboratorios virtuales. Revista Electrónica de Enseñanza de las Ciencias, 6(3), 562-576.

Márquez-Bargalló, C., \& Roca-Tort, M. (2006). Plantear preguntas: Un punto de partida para aprender ciencias. Revista Educación y Pedagogía, 18(45), 63-71.

Nérici, I. G. (1969). Hacia una didáctica general dinámica. Biblioteca de Cultura Pedagógica, Serie Didáctica. Editorial Kapelusz, Buenos Aires, Argentina.

Paniagua, M. E. (2004). La formación y la actualización de los docentes: Herramientas para el cambio en la educación. En Centros de Estudios Democráticos de América Latina (CEDAL) (Ed.), Dimensiones de la educación en Costa Rica. San José, Costa Rica.

Pereira-Chaves, J. (Setiembre 2010). La enseñanza de la biología en Costa Rica: Los retos del abordaje pedagógico para la alfabetización científica y la motivación estudiantil en secundaria. En M. I. Vollmer (Presidencia), Congreso Iberoamericano de Educación, METAS 2021. Congreso llevado a cabo en Buenos Aires, Argentina.

Pereira-Chaves, J., Camacho-Vargas, S., \& Muñoz-Simon, N. (2013). La Olimpiada Costarricense de Ciencias Biológicas y su papel en la comunidad educativa nacional mediante la integración del competidor, compartir, convivir y aprender. Revista Uniciencia, 27(1), 245-265. 
ISSN Electrónico: 2215-3470

DOI: http://dx.doi.org/10.15359/ru.31-2.6
UNICIENCIA Vol. 31, No. 2, pp. 83-97. Julio-diciembre, 2017.

URL: www.revistas.una.ac.cr/uniciencia Email: revistauniciencia@una.cr

Quinquer, D. (2004). Estrategias metodológicas para enseñar y aprender ciencias sociales: Interacción, cooperación y participación. Íber, 40, 7-22.

Ramírez-Silva, E., Addine-Fernández, R., \& Addine-Ramírez, B. (2010). La prensa escrita como alternativa para incentivar la cultura desde la enseñanza de la biología. Revista Didasc@lia: Didáctica y Educación, 4, 47-58.

Rodríguez-Calvo, M. (2012). Olimpiadas Costarricenses de Ciencias Biológicas como proceso fundamental en el mejoramiento de la calidad educativa secundaria de Costa Rica en el área de la biología. Revista Digital Calidad en la Educación Superior, VI Edición, 3(2), 122-140.

Rodríguez-Calvo, M., \& Pereira-Chaves, J. (2015). Unión interuniversitaria para el fortalecimiento de las Olimpiadas Costarricenses de Ciencias Biológicas como proceso de formación de los futuros profesionales en el área de las ciencias y la actualización de los conocimientos de los y las docentes de secundaria sobre biología. Revista Electrónica Calidad en la Educación Superior, XI Edición, 6(1), 91-118.

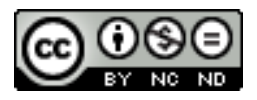

Caracterización de los profesores de biología participantes en la VII Olimpiada Costarricense de Ciencias Biológicas: una mirada de sus estudiantes competidores (Federico Herrera-Madrigal y otros.) por Revista Uniciencia se encuentra bajo una Licencia CreativeCommons Atribución-NoComercial-SinDerivadas 3.0 Unported. 